\title{
Des biens communs aux biens publics mondiaux
}

\section{Bernard Hours}

\section{(2) OpenEdition \\ Journals}

Édition électronique

URL : http://journals.openedition.org/developpementdurable/5613

DOI : 10.4000/developpementdurable.5613

ISSN : 1772-9971

Éditeur

Association DD\&T

\section{Référence électronique}

Bernard Hours, "Des biens communs aux biens publics mondiaux », Développement durable et

territoires [En ligne], Points de vue (2003-2010), mis en ligne le 07 mars 2008, consulté le 21 avril 2019

URL : http://journals.openedition.org/developpementdurable/5613 ; DOI : 10.4000/

developpementdurable.5613

Ce document a été généré automatiquement le 21 avril 2019

\section{(c) (1) 8}

Développement Durable et Territoires est mis à disposition selon les termes de la licence Creative Commons Attribution - Pas d'Utilisation Commerciale 4.0 International. 


\title{
Des biens communs aux biens publics mondiaux
}

\author{
Bernard Hours
}

1 La notion de « biens communs » est investie d'une portée particulière à une époque ou on utilise aussi le concept de "biens publics mondiaux" sorti tout droit des divers processus plurivoques qui constituent la globalisation aujourd'hui. Ces expressions, proches mais différentes, amènent nécessairement à s'interroger, moins sur la propriété que sur l'appropriation et ses différentes formes. Elles appellent aussi une réflexion sur la production de la valeur et la rareté.

2 Cet article rappelle d'abord les principales approches anthropologiques sur la circulation des biens, les échanges, l'appropriation. La notion de biens communs renvoie à un âge d'or antérieur à la propriété, une sorte de paradis perdu largement mythique. On s'interroge ensuite sur la notion actuelle de biens publics mondiaux, qui vient sublimer l'enfer de l'exploitation et de la concurrence à l'échelle globale.

3 Dans une première partie on se penchera d'un point de vue général sur la propriété telle qu'elle est abordée par l'anthropologie académique. On analysera ensuite les "biens communs" à l'épreuve de l'anthropologie, ou de certaines de ses tendances. On traitera enfin de l'actualité de cette notion dans ses différentes dimensions, c'est-à-dire de sa pertinence au temps de la globalisation à travers le concept de biens publics mondiaux.

1. Anthropologie et propriété

4 Suivant Levi Strauss (2002), les sociétés sont fondées sur des règles dont celles qui régissent la parenté. Ces dernières visent à assurer la reproduction sociale à travers, en particulier, des femmes. Parmi les femmes, il faut distinguer celles qui sont proscrites pour risque d'inceste, et celles qui sont accessibles, voire recommandées (les mariages entre cousins croisés). L'entre soi, comme l'autre, sont présents dans ces distinctions. Entre soi se thésaurisent les "héritages" de toutes natures, les "patrimoines". Vers l'autre s'ouvrent les échanges, avec leurs risques et inconnues.

5 Les alliances (mariages) créent des liens qui produisent la société comme lien de ces liens, à travers l'observance de règles de conduites prescrites dont les règles de parenté sont la matrice et le modèle. Outre ces flux d'épouses échangées suivant des règles de circulation 
strictes d'autres types de biens sont échangés. Pour qu'une personne ou un objet devienne un bien il faut qu'il soit porteur d'une valeur d'usage et d'échange, à la fois. Les biens se réfèrent aussi au bien de façon latente mais forte. Ce bien peut être la capacité d'engendrer des enfants pour une femme, ou la nourriture accessible ou produite par un outil. De nombreux biens peuvent être abordés dans une logique utilitariste. Mais qu'en est-il de ceux dont l'utilité primaire, la fonction pratique, n'est pas évidente ? C'est ici que s'insère toute la réflexion anthropologique sur l'échange, le don, la réciprocité, qui fascine légitimement les économistes, habitués à des transactions terme à terme, à des réciprocités à cycles courts, à de supposés équilibres de valeurs relativement statiques.

Si l'échange des femmes est le modèle de l'échange entre deux entités sociales exogames, d'autres valeurs circulent et sont échangées dans les sociétés dites "traditionnelles". La Kula des trobiandais de Mélanésie décrite par Malinowsky (1974) est exemplaire à cet égard. Elle met en œuvre des cycles d'échanges de longue durée de portée non plus locale mais régionale, entre des îles distantes. Les deux formes d'objets échangés (coquillages et haches) circulent selon deux cycles, dans deux sens symétriques. La valeur de ces objets est symbolique et rituelle. C'est une valeur d'échange. C'est pourquoi les économistes n'ont pas manqué d'y voir une "monnaie primitive", à ceci près que sa théorisation est culturellement impensable puisque c'est une valeur qui circule en permanence et doit circuler en permanence au risque de bloquer le système et de perdre toute dignité statutaire pour celui qui refuserait de participer au cycle avec ses partenaires prescrits, suivant le calendrier prévu et lîle concernée. La Kula souligne l'importance sociale de la réciprocité dans les cycles d'échanges qui produisent les liens sociaux et politiques entre sociétés segmentaires sans Etat. La réciprocité produit dans une large mesure la valeur des biens échangés qui ne sont pas des biens destinés à un usage prosaïque ou à une vulgaire consommation, mais des biens à forte valeur symbolique ajoutée qui peuvent être accumulés mais non thésaurisés. Ils ne doivent pas cesser de circuler sinon ils perdent toute valeur.

7 Tout échange suppose et produit des relations sociales et la réciprocité est la clé de la pérennité de ces relations sociales. Face à la force de la réciprocité qui produit la confiance trône le don qui fascine anthropologues et économistes depuis les travaux de Marcel Mauss (1966). Le don surplombe de manière énigmatique tout le champ d'une virtuelle anthropologie économique. Le don serait la subversion de l'économie de marché et de la mesquinerie comptable car il participe d'une dilapidation volontaire de richesse produisant du prestige capitalisé en statut. Le potlach analysé par Mauss est un acte agonistique d'accumulation de biens en vue de leur destruction ostentatoire et publique. Nous ne sommes plus dans un échange entre acteurs virtuellement égaux mais dans une affirmation concurrentielle de prestige produit par la destruction des biens et non leur circulation, encore moins leur distribution. Il s'agit de la plus radicale contestation de l'économie pensable puisque qu'il s'agit de la fin ou de l'interdiction de tout échange ultérieur. Ce don n'est destiné à personne. Il s'autodétruit. Les cérémonies, souvent funéraires, où sont brûlés des billets de banque sont fréquentes dans plusieurs sociétés, asiatiques en particulier. Le potlatch pose radicalement le problème de l'accumulation qui s'oppose à l'échange, sauf dans le capitalisme où l'accumulation du capital se nourrit de l'échange des biens sur le marché. Dans l'économie capitaliste l'accumulation a pour objectif l'accumulation, avec une faible production de statut, puisque, s'il y a bien un certain prestige accolé à la figure des capitaines d'industrie ou des "golden boys" post modernes, ils sont l'objet d'un égale réprobation de la part d'une autre partie de leur 
société. Ce n'est que lorsque Bill Gates et Waren Buffet se dépossèdent ostentatoirement d'une petite partie de leur fortune au profit de fondations humanitaires - pour des raisons largement fiscales - qu'il y a production de prestige et de statut, à la façon de big men mélanésiens. Leur stratégie reste néanmoins très éloignée de celle de la destruction de richesse dans le potlatch, conduite impensable en termes de rationalité économique, le postulat fondateur de l'économie politique.

8 Le don fascinera durablement les économistes comme les kamikazes terrassent et fascinent les Occidentaux si attachés à la vie biologique. Une économie de dilapidation n'est pas pour demain car elle est interdite tant que la pauvreté et le manque frappent la majorité des sociétés. L'essai sur le don de Mauss évoque des "sociétés d'abondance" (cf. supra) ou de faible rareté, ou encore d'inégalités statutaires plus qu'économiques. Il n'y a de dilapidation ostentatoire possible que dans des sociétés où le manque est partagé et de faible amplitude, sinon la société explose car le lien social est rompu. Seules des dignités et des indignités statutaires établies peuvent permettre "un certain" consentement à la misère, acceptable comme effet du statut ( $c f$. les intouchables en Inde) mais inacceptable dans le cadre d'une démocratie à vocation égalitaire en droit et d'une économie de marché.

Il parait évident que c'est la rareté, lorsqu'elle est appréhendée hors de ses éventuels déterminants statutaires fondant les hiérarchies sociales, qui introduit la valeur marchande des biens comme référent social et monétaire. C'est ainsi que les biens deviennent des marchandises qui s'échangent sur un marché basé sur la concurrence, la manipulation des besoins en amont afin de justifier l'offre et de créer en aval une demande. L'économie politique peut alors raisonner.

10 L'anti utilitarisme cher à la Revue du MAUSS et à Alain Caillé fasciné par le don (Caillé, 2007), se situe dans ce contexte, celui d'une réaction salutaire mais exposée à réifier la notion de don pour en faire un archétype de conduite alors qu'il s'agit, d'abord, d'une pratique sociale productrice d'intégration sociale appuyée sur le principe de réciprocité.

11 A la lecture de ces approches anthropologiques, il est assez compréhensible d'y voir en filigrane une réprobation virtuelle à l'égard de la propriété. Celle-ci apparaitrait comme le résultat d'accaparements et d'abus qui auraient mis un terme à "un âge d'or " pré marchand et pré capitaliste. Cette vision irénique est un peu réductrice. Elle occulte le fait que certains biens ont été appropriés depuis des lustres, les femmes en particulier, qui sont affectées exclusivement à leurs époux, à l'exception de quelques groupes aussi évoqués qu'ils sont peu nombreux. Il reste néanmoins que l'appropriation des biens résulte, dans une large mesure, de la modification ou du détournement de flux de circulation établis qui sont peu à peu détournés de leur logique fondatrice de production de biens réciproques. C'est toute la question, abondamment documentée, de l'évolution des systèmes économiques, à laquelle Polanyi (1983) a apporté une contribution majeure voire essentielle.

2. Les biens communs à l'épreuve de l'anthropologie

12 L'ouvrage de Marshall Sahlins Age de pierre, âge d'abondance (1976) est le prototype des analyses d'un âge d'or pré marchand dont on n'a jamais cessé de débattre pour y trouver les parts respectives des phantasmes et de la réalité. Sahlins développe l'idée que dans de nombreuses sociétés dites traditionnelles la charge de travail est modérée car la nature fournit, moyennant des efforts limités, des moyens de subsistance relativement abondants sous formes de fruits, légumes, gibiers. Se télescopent ici le «bon sauvage » et «l'âge d'or ». L'abondance de ces ressources (naturelles) tend à produire, selon P. Clastres 
(1974), des sociétés sans Etats, peu inégalitaires. L'Etat, selon lui, développe ou crée les inégalités. Formulées dans les années 1970, ces approches participent d'une lecture depuis lors très contestée des sociétés non marchandes. Elles s'inscrivent dans l'anti productivisme postérieur au mouvement de 1968 qui fut utopique et culturaliste.

Il faut néanmoins retenir que le travail peut être, en partie, déconnecté de la production de marchandises et que celles-ci n'apparaissent que lorsque elles peuvent s'inscrire dans des marchés où s'échangent des objets de valeur et non plus seulement des valeurs symboliques hors utilité.

Les « bons sauvages » évoqués ressemblent fort à Robinson Crusoé à ceci près qu'ils vivent en société et échangent des femmes, des prestations virtuelles. C'est l'absence de rareté et la faible quantité de travail nécessaire à la survie qui rendraient communs des biens pré marchands. Et c'est cette même rareté qui introduit la valeur marchande de ces biens. On comprend que dans ce cadre la taille des unités sociales soit déterminante pour raisonner. L'augmentation de la population, sa concentration, produisent des changements économiques d'échelles et de modes d'organisation politique et sociale.

Si les sociétés pré marchandes montrent l'existence de produits sans valeur car disponibles et dépourvus de rareté, ces denrées ou objet sont sans valeur d'échange et ne sont pas des biens mais une partie de l'environnement naturel pour ce qui concerne par exemple les fruits. A l'inverse, dès qu'il y a plantation et récole nous sommes en présence de biens, antichambre de la marchandise puisqu'il y a création de valeur d'échange, au moins potentielle.

Les fameux « naturels » du XVII siècle reviennent désormais au galop... là où ils n'étaient pas attendus. En effet, on constate aujourd'hui la montée en puissance de l'idée selon laquelle la nature (celle de l'environnement) est une richesse rare, qu'elle recèle des ressources peu renouvelables, rares elles aussi, que sa protection est devenue une question de survie d'abord, puis de vie durable.

17 Nous voilà face au développement durable qui se préoccupe, enfin, de la pérennité des échanges marchands portant sur des marchandises rares et peu renouvelables à la fois.

Ce monde du développement durable est à l'antithèse de celui des bons sauvages de l'âge d'or, supposés dépourvus d'inquiétudes et de soucis de gestion, ce qui est évidemment inexact, puisqu'ils avaient d'autres soucis tels que la maladie, les cataclysmes... Le principe de précaution sert la durabilité du "développement" mais le bon sauvage ne manquait pas de prudence non plus face aux dangers de la chasse.

19 Au-delà des distinctions classiques à propos de la notion de biens communs, l'anthropologie a surtout mis en lumière les logiques à l'œuvre dans les sociétés pré marchandes et pré étatiques. Il est difficile d'échapper à une lecture non mythique de ces sociétés pour la plupart éteintes où la rareté ne pesait pas, ou peu, sur la valeur et où la sphère des échanges, fréquemment cérémoniels, avait plus d'importance et de valeur (sociale) que celle des biens, devenus depuis marchandises.

20 L'anthropologue doit s'interroger sur le terme «communs » accolé aux biens. A quelle "communauté » se réfère-t-il ? Quel partage présume-t-il ? Commun suppose non seulement un partage mais un partage égalitaire, moins en réalité que comme accès virtuel. En effet chacun peut ramasser des fruits selon ses besoins mais dès qu'apparaît la rareté s'imposent les contraintes liées à la valeur.

21 K. Polanyi a apporté des éléments essentiels pour éclairer ces domaines. Avec d'autres auteurs, il faut revenir à la question de l'appropriation de la terre en Angleterre à la fin 
du Moyen âge. La mise en place du système des « enclosure » constitue bien une révolution largement irréversible en Occident. En clôturant des champs auparavant communs, bien que régis par des droits spécifiques, là propriété foncière a pu s'établir et se développer. La terre devient un bien rare et acquiert une valeur qui entretient sa rareté. On sait qu'il faut y voir la naissance d'une bourgeoisie rurale avant d'être urbaine. Avant ces "enclosure " les terres appartenaient à des seigneurs qui en concédaient l'usage à "leurs" paysans redevables d'une taxe en nature. Un tel système, sans être similaire, n'est pas très éloigné des régimes observés en Inde ou en Chine. Le contrôle sur la terre découle d'un statut social qui le légitime comme il légitime la captation d'une partie des surplus des paysans. Viennent les guerres qui opposent ces potentats locaux et la nécessité de capitaliser les ressources en hommes, armes et nourriture. Les vainqueurs deviennent princes et rois.

Ces évocations rapides amènent à souligner la rupture introduite par l'appropriation privée de la terre qui marque le début de la gestion des biens rares dont la notion perdure jusqu'à nous. On comprend l'importance de questions latérales telles que la charge de population sur un territoire qui accentue l'émergence de la rareté. Vient un moment où ce qu'offre la nature ne suffit plus et il devient nécessaire de développer les capacités de cette nature à travers des évolutions techniques et des changements de rapports dans la société, en particulier en termes de modes de gestion de la force de travail et de statut social des travailleurs. Le travail devient une valeur ajoutée à celle de la terre et ultérieurement une valeur marchande échangeable.

On pourrait considérer que nous sommes aujourd'hui au terme de ce processus long de plusieurs siècles, occidental d'abord, mais pertinent ailleurs aussi sous des formes diverses. L'exploitation de la nature et son appropriation ont atteint une cote d'alerte exprimée par l'inquiétude environnementale. Certes l'environnement n'est plus la nature des «bons sauvages", ni un paysage tropical luxuriant. C'est un concept construit et en partie abstrait qui met en avant l'émergence de contraintes spécifiques techniquement (ex. la pollution de l'eau ou de l'air) mais tellement partagées dans différentes sociétés qu'elles en deviennent "communes", tant le discours environnementaliste oppose durable et précaire et renvoie à une communauté d'intérêts ou de survie selon des modes plus ou moins catastrophistes mais toujours alarmistes comme si l'exploitation déclenchée avec les «enclosures» atteignait aujourd'hui une butée à la fois quantitative et qualitative. C'est pourquoi, au-delà des biens communs, sont apparus les "biens publics mondiaux", version globalisée des biens communs.

3. Des biens communs aux biens publics mondiaux

24 Faire référence à des biens "communs" présume de l'existence d'une communauté sociale et donc d'une appartenance à un groupe identifié et circonscrit. Les serfs d'un seigneur ou d'un zamindar, indien, les citoyens d'une république, les habitants de la planète, peuvent se référer à une entité politique (sauf la planète) de taille très variable dont ils sont membres, ce qui leur confère des droits d'accès, de jouissances, ou d'exploitation.

L'anthropologue, parce qu'il était familier de sociétés pré marchandes, sait que la rationalité économique est une construction historique datée dont la cohérence est aussi forte qu'elle est relative. Les sociétés concrètes ne sont pas peuplées d'hommes abstraits mais d'acteurs sociaux statutairement identifiés.

La consommation de masse produit en permanence de la différenciation autant que du semblable. Mais les registres de différenciation et de similitude varient d'une époque à l'autre. Dans nos sociétés post industrielles où sévissent le chômage et la pauvreté, pour 
certains, l'appartenance et l'identification à un groupe social sont extrêmement fragilisées. L'argent apparaît comme le principal instrument d'intégration et de production de statut. A l'inverse, son manque provoque l'exclusion.

Dès lors qu'une économie de marché mondialisée tend à se mettre en place les droits sociaux gagnés au XXe siècle par des luttes sociales sont fragilisés et régressent sous le coup d'un nivellement par le bas tendanciel. En contre partie - si l'on peut dire apparaissent des droits de l'espèce humaine appelés droits humains, probablement pour s'écarter de l'origine révolutionnaire des droits de l'homme. L'homme est un sujet de l'histoire. L'humain est une espèce biologique, naturelle comme les grenouilles ou les bébés phoques. Aux côtés d'une globalisation économique, par l'économie de marché mondialisée, apparaît une globalisation éthique appuyée sur des droits humains et une limitation (abstraite et théorique) de l'exploitation, celle des enfants et des femmes, ou d'autres catégories identifiables. A quelle communauté renvoient ces droits globaux, globalement distribués, comme un chèque en blanc sur une banque qui n'existe pas, ou pas encore? La seule communauté intelligible derrière ces droits c'est celle de l'espèce humaine sur sa planète divisée par des frontières, des langues, des religions, des Etats, des intérêts concurrents attisés par l'économie de marché et la course aux profits financiers.

Les risques environnementaux mettent en lumière des biens communs naturels, attributs de la planète. La prise de conscience d'une entité naturelle globale est ici nouvelle et manifeste. Mais la plupart des contraintes environnementales s'opposent à une exploitation sans mesure des ressources et donc à de meilleurs profits. Face à cette contradiction imparable le gain à court terme l'emporte pour le moment. Mais l'apparition de la référence à des «biens public mondiaux " vient remettre en vue la nécessité d'une domestication de la course aux profits, sans règles ni moral. Les biens publics mondiaux se présentent donc comme des biens communs globalisés. Si ces biens communs demeurent dans la sphère de l'économie, ils sont incompatibles avec le capitalisme et la concurrence. Mais l'existence de biens communs moraux, appelés droits humains, vient pour nous rassurer, bien qu'aucune instance juridique ne soit en mesure de faire entériner ces droits par une "force publique" qui n'existe pas. Le patrimoine de l'UNESCO met de son côté en scène des biens communs esthétiques et tente d'en faire une valeur déterritorialisée par le recours à la notion de biens publics mondiaux qui s'impose si ce patrimoine est celui de l'humanité.

La santé est en voie de globalisation rapide à travers les efforts de gestion transnationale des épidémies et la veille épidémiologique planétaire. Le régime politique chinois est tolérable mais l'absence de maîtrise des risques épidémiologiques serait intolérable. Les Chinois l'ont bien compris. La santé publique peut donc être considérée comme un bien public mondial en voie de construction. Il y a certes tous ceux qui sont loin derrière ... mais tous les Etats qui disposent de ressources financières et techniques en la matière sont engagés. C'est ici qu'il apparaît avec clarté que si la santé est virtuellement un bien public mondial alors qu'elle n'est nullement un bien commun, c'est que le concept de biens publics mondiaux permet d'occulter les contraintes des inégalités planétaires en les sublimant dans une sorte d'espéranto idéologique où tout n'est que luxe, calme et volupté planétaires.

Des biens communs avant les "enclosure» aux biens publics mondiaux d'aujourd'hui circule la question de l'appartenance des hommes à une entité ou communauté politique et sociale. Les rapports économiques aujourd'hui privilégiés ne produisent pas de liens sociaux, juste des échanges marchands, avec parfois un voisinage des plus étroits avec la 
délinquance financière. Parce que les biens communs ont - de fait - quasiment disparus sous les coups de l'économie marchande, l'apparition des biens publics mondiaux vient heureusement inscrire un objectif moins grossièrement prédateur que les agissements des raiders et traders.

Cette apparition appelle une question conséquente sur la société civile mondiale virtuelle que présume toute référence à des biens publics mondiaux. Mise à part l'identité naturelle de la planète comme "notre environnement ", à quelle entité se réfèrent les biens publics mondiaux pour être publics et mondiaux? Mondiaux, ils le sont, car ils nourrissent l'idée d'un monde virtuellement unifié par les divers processus de globalisation.

publics, le sont-ils? Et au nom de quelle collectivité, sinon celle de l'espèce humaine? Mais quel est l'espace politique et social où s'inscrit cette espèce ? Force est de constater qu'elle est déterritorialisé, délocalisée, comme les usines. On est ainsi amené à se demander si ces biens publics mondiaux virtuels sont un leurre destiné à calmer nos inquiétudes face à la sauvagerie des marchés ou un projet digne de ce nom dont on aurait simplement oublié de penser la mise en œuvre ou le mode d'emploi.

Peut-être s'agit-il simplement d'un jeu vidéo ou tout devient possible sans le moindre effort sur le réel. De tous les " biens publics mondiaux », l'environnement et la santé sont certainement ceux qui ont la plus grande chance de survie et de développement durable. La question est donc de savoir dans quels délais les marchands de $\mathrm{CO}^{2}$ et de médicaments baisseront un peu les bras.

Les biens communs nous rappellent un monde pré capitaliste et pré marchand, la nature avant la rareté, à laquelle on prête évidemment toutes les vertus. Les biens publics mondiaux sont un concept postmoderne d'humanisation du capitalisme global, en particulier à partir de l'environnement en crise et de la santé. C'est l'espèce humaine en danger qui est exposée en tant que concept abstrait soumis à la gestion des risques et non plus au partage de ressources communes. Les biens ont cessé d'être communs. Ce sont les risques qui sont partagés.

\section{BIBLIOGRAPHIE}

Caillé A. (2007) Anthropologie du bien. La découverte.

Clastres P. (1974) La société contre l'Etat, Editions de Minuit, Paris.

Levi Stauss Cl. (2002) Les structures élémentaires de la parenté. Editions de l'EHESS.

Malinowsky B. (1974) Argonautes du pacifique occidental, Gallimard, Paris.

Mauss M. (1966) Sociologie et anthropologie, PUF, Paris.

Polanyi K. (1983) La grande transformation, Gallimard, Paris.

Sahlins M. (1976) Age de pierre, âge d'abondance. L'économie des sociétés primitives, Gallimard, Paris. 


\section{RÉSUMÉS}

La notion de « biens communs » est investie d'une portée particulière à une époque ou on utilise aussi le concept de "biens publics mondiaux" sorti tout droit des divers processus plurivoques qui constituent la globalisation aujourd'hui. Ces expressions, proches mais différentes, amènent nécessairement à s'interroger, moins sur la propriété que sur l'appropriation et ses différentes formes. Elles appellent aussi une réflexion sur la production de la valeur et la rareté. Cet article rappelle d'abord les principales approches anthropologiques sur la circulation des biens, les échanges, l'appropriation. La notion de biens communs renvoie à un âge d'or antérieur à la propriété, une sorte de paradis perdu largement mythique. On s'interroge ensuite sur la notion actuelle de biens publics mondiaux, qui vient sublimer l'enfer de l'exploitation et de la concurrence à l'échelle globale.

The notion of common good has a specific role at the present time of globalisation. The concept of global public good is also used in this context. These terms, close together but also different, bring to the questions of appropriation, production and scarcity. This article first reminds the main anthropological approaches on circulation of goods, exchanges, and appropriation. The notion of common goods makes a reference to a golden age, a lost paradise before property. Then we propose a critical analysis of global public goods which are supposed to sublimate global exploitation and competition.

\section{INDEX}

Mots-clés : biens communs, propriété, biens publics mondiaux, anthropologie

Keywords : Common goods, property, globalisation, global public goods, anthropology

\section{AUTEUR}

\section{BERNARD HOURS}

Bernard Hours est anthropologue, Directeur de recherche à l'Institut de Recherche sur le Développement. Ses travaux récents portent sur les questions sécuritaires et la globalisation. 